\title{
Würdigung medizinischer Durchbrüche
}

Max Geiser

Korrespondenz: Prof. Dr. med. Max Geiser Gossetstrasse 49 CH-3084 Wabern Tel. 0319611639
Das Ende des 19. und das 20. Jahrhundert brachten medizinische Durchbrüche mit einer grossen Verbesserung der Lebensumstände für die ganze Bevölkerung in den Industrieländern, unabhängig vom sozialen Status. Die Durchbrüche betrafen viele Infektionskrankheiten und Seuchen [1] wie Pest, Pocken, Cholera, Milzbrand, Tuberkulose, Lepra, Diphtherie, Typhus, Wundinfektionen (Gasbrand, Tetanus, Sepsis und Kindbettfieber), Geschlechtskrankheiten, Hepatitis, Poliomyelitis und Malaria. Tollwut und Vitaminmangelkrankheiten wie Skorbut, Rachitis und Beriberi verschwanden. Struma und Karies wurden dank Jod und Fluor in die Schranken gewiesen. Die Reparaturmedizin machte bei Herz-, Lungen-, Nieren-, Gefässund Gelenkskrankheiten sowie angeborenen Krankheiten und Missbildungen willkommene Fortschritte.

Angesichts der in der kurzen Zeit von 150 Jahren erbrachten Leistungen der naturwissenschaftlich fundierten Medizin, die nach den Ursachen von Krank- druck der Marken nicht erlaubt.) Die Postmarken würdigen die Entdeckung der Übertragung des Malariaerregers durch Moskitos 1897 dank Sir Ronald Ross (Nobelpreisträger 1902), die Entdeckung der antibiotischen Eigenschaften des Penicillins durch den Mikrobiologen Sir Alexander Fleming 1928 (Nobelpreis 1945), die Einführung der Implantationschirurgie von Plastiklinsen durch Sir Harold Ridley 1949, die Synthetisierung von Betablockern gegen Hypertension und Angina pectoris durch Sir James Black 1962, die bahnbrechende Erfindung des künstlichen Hüftgelenksersatzes durch den orthopädischen Chirurgen Sir John Charnley 1962 und die Erfindung der Computertomographie durch Sir Godfrey Hounsfield 1971 (Nobelpreis 1979.)

Die Markenserie zeigt beispielhaft, dass die Ärzte im 20. Jahrhundert dank technologischen und naturwissenschaftlichen Durchbrüchen den bemerkenswerten Ruf als Experten erlangten. Sie trugen in

\section{Die Markenserie der Königlichen Britischen Münzanstalt mahnt die Öffentlichkeit an die Notwendigkeit naturwissenschaftlicher Forschung.}

heiten und Verletzungen sucht und diese eliminieren will, sind weitere Erfolge zu erwarten, am wünschenswertesten bei der Verhütung und Heilung von Tumorkrankheiten und der noch zu wenig erforschten Hirnkrankheiten (Morbus Bleuler, Alzheimer und Parkinson), bei denen bis heute keine Heilung gefunden wurde.

\section{Würdigt die Öffentlichkeit die medizinischen Durchbrüche?}

Der heutigen Bevölkerung ist kaum bewusst, dass die Heilkunde während Jahrtausenden bis ins 19. Jahrhundert hinein von dogmatischen Lehrmeinungen beherrscht war und, abgesehen von der samariterlichen Zuwendung und Pflege, ernsthaften Krankheiten und Verletzungen praktisch hilflos gegenüberstand. Weil die durch die naturwissenschaftlich fundierte moderne Medizin herbeigeführte Verbesserung der Condition humaine von der Öffentlichkeit mehrheitlich als selbstverständlich, wie vom Himmel gefallen, wahrgenommen wird, ist es sehr erfreulich, dass auf der kürzlich von der Königlichen Britischen Münzanstalt herausgegebenen Markenserie sechs britische Forscher gewürdigt werden, denen verschiedene «Medical breakthroughs» im 20. Jahrhundert zu verdanken sind. (Leider hat die Royal Mail den Ab-
Grossbritannien und anderen die naturwissenschaftliche Forschung pflegenden Ländern entscheidend zum Vertrauensverhältnis zwischen Patient und Arzt

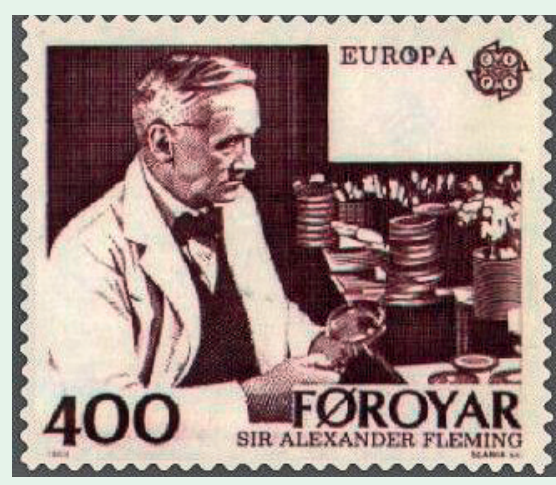

Dies ist keine Briefmarke der im Text erwähnten Serie der englischen Post, sondern eine alte Marke der Färöer-Inseln. Die «Royal-Mail» hat den Abdruck ihrer Marken-Serie in der SÄZ leider nicht genehmigt. Auch eine ihrer Marken zeigt, so wie die hier abgebildete, Sir Alexander Fleming, der die antibiotischen Eigenschaften des Penicillins entdeckte. Zu sehen sind die englischen Marken unter www.royalmail.com/portal/stamps $\rightarrow$ Past stamps $\rightarrow 2010 \rightarrow$ Medical Breakthroughs. 
bei, weil sie dank der wissenschaftlich erarbeiteten Wirksamkeit der Krankheits- und Verletzungsbehandlung die Verantwortung für die Gesundheitsfürsorge der ganzen Bevölkerung unabhängig vom sozialen Status des Patienten übernehmen konnten. Damit wurde die Sorge um die Gesundheit ein Grundrecht der Bürgerin und des Bürgers im modernen Staat, wobei von aufgeschlossenen Bürgerinnen und Bürgern erwartet wird, dass sie sich verantwortungsvoll um die Erhaltung ihrer Gesundheit kümmern.

\section{Die Wiederbelebung der Quacksalberei}

Aus dem Beitrag «Welche Therapie darf's denn sein?» im «Bund» vom 6.1.2011 geht hervor, dass in der Schweiz gemäss dem erfahrungsmedizinischen Register EMR 17200 meist nicht-ärztliche Alternativ- und Komplementärtherapeuten tätig sind, die eine der

\section{Keiner einzigen der über 100 alternativen Praktiken} ist je ein medizinischer Durchbruch gelungen.

125 Praktiken anbieten. Angeblich rechnen viele Ärzte ihre Alternativ- und Komplementärangebote via solidarisch finanzierte Grundversicherung ab. Bekanntlich wurde bei einer Volksabstimmung die Berücksichtigung der Komplementärmedizin in der Bundesverfassung verankert. Und der Ständerat hat am 9.3.2010 eine Motion seiner Wissenschaftskommission gutgeheissen, die die Ausbildung von Ärzten, Zahnärzten, Apothekern und Chiropraktoren in Alternativmedizin fordert und von den Universitäten die Abhaltung von Kursen in Komplementärmedizin verlangt. Die Mehrheit der Politiker hat offensichtlich aus der Geschichte der Heilkunde nichts gelernt und nicht zur Kenntnis genommen, dass keiner einzigen der über 100 alternativen Praktiken je ein medizinischer Durchbruch gelang. Der Bei-

\footnotetext{
Standpunkt - Point de vue

Die in der Rubrik «Standpunkt» veröffentlichten Beiträge bringen die persönliche Meinung des Autors zum Ausdruck, unabhängig von den Haltungen und Ansichten der Redaktion und der FMH.

Les articles publiés sous «Point de vue» représentent l'opinion de leur auteur, indépendamment de la position de la rédaction ou de celle de la FMH.
}

trag dieser zur Zeit Hochkonjunktur geniessenden Praktiken besteht lediglich in der unnötigen Aufblähung des Gesundheitsmarktes mit Quacksalbereien und in der Irreführung und Täuschung gutgläubiger Menschen mit Plazebos, die im Falle einer möglichen wirksamen Behandlung diese verzögern oder verhindern und damit Patienten schädigen [2-7].

\section{Schlussfolgerungen}

Die Würdigung einiger medizinischer Durchbrüche seit dem Ende des 19. Jahrhunderts, die naturwissenschaftlich nach Krankheits- und Verletzungsursachen suchenden Forschern und technologischen Pionieren zu verdanken sind, ist sehr erfreulich. Die Markenserie der Königlichen Britischen Münzanstalt mahnt die Öffentlichkeit an die Notwendigkeit naturwissenschaftlicher Forschung, die allen Menschen mehr nützt als politische Wichtigtuerei. Vielleicht entschliesst sich die Schweizer Post oder die Swissmint früher oder später dazu, einen oder mehrere Nobelpreisträger für Medizin auf einer Postmarke oder Banknote zu würdigen. Es könnten seit Theodor Kocher bis und mit Rolf Zinkernagel sechs weitere Schweizer Träger des Nobelpreises für Medizin auf diese Weise für die an den medizinischen Grundlagenwissenschaftern nicht sonderlich interessierte Öffentlichkeit sichtbar gemacht werden.

Trotz der bahnbrechenden Erfolge der im 19. Jahrhundert revolutionierten Medizin sind auf dem helvetischen Gesundheitsmarkt mehr als 17000 Alternativ- und Komplementärtherapeuten tätig und bieten über 100 verschiedene, angeblich heilende Praktiken an, ohne bis heute einen einzigen allen Menschen dienenden medizinischen Durchbruch vorweisen zu können.

\section{Literatur}

1 Winkle S. Geisseln der Menschheit. Kulturgeschichte der Seuchen. Mannheim: Artemis und Winkler; 2005.

2 Thomas L. On magic in medicine in «The Medusa and the Snail». New York: The Viking Press; 1979.

3 Clark G, Stalker D. Engineers, cranks, physicians, magicians. N Engl J Med. 1983;308:960.

4 Skrabanek P, McCormick. Follies and Fallacies in Medicine. Glasgow: The Tarragon Press; 1989.

5 Abgrall JM. Les charlatans de la santé. Paris: Documents Payot; 1998.

6 Geiser M. Der menschliche Körper - die letzte Bastion der Magie. Schweiz Ärztezeitung. 1988;69:113.

7 Ernst E. The Desktop Guide to Complementary and Alternative Medicine. Edinburgh, London, New York: Mosby; 2001. 